$\checkmark$ Research Square

\title{
Measuring flavin mononucleotide concentrations in whole blood, red cell based or acellular perfusate samples by fluorescence spectrometry
}

Tine Wylin

KU Leuven

Veerle Heedfeld ( $\nabla$ veerle.heedfeld@kuleuven.be )

KU Leuven

Ina Jochmans

KU Leuven https://orcid.org/0000-0003-4592-2810

\section{Method Article}

Keywords: Flavin mononucleotide, fluorescence spectrometry

DOl: https://doi.org/10.21203/rs.3.pex-1536/v1

License: (c) (i) This work is licensed under a Creative Commons Attribution 4.0 International License.

Read Full License 


\section{Abstract}

Flavin mononucleotide (FMN) is non-covalently bound to complex I of the mitochondrial respiratory chain. During ischemia-reperfusion injury, reduced FMN is released from complex I, leaving the complex impaired. In literature, FMN measured during ex situ organ perfusion has been considered as a biomarker for organ graft quality. With this protocol the FMN concentrations in perfusate samples taken during organ perfusion can be estimated quickly and easily by fluorescence spectrometry. The use of nonbinding plates is essential. A fresh standard curve using riboflavin 5'-monophosphate sodium salt hydrate must be prepared every day. To quantitatively compare sample concentrations between different plates, it is advisable to include an internal standard. This internal standard is best prepared by pooling samples from experiments that use the same perfusate composition. This assay will work for samples acquired from whole blood, red cell based solutions or acellular solutions.

\section{Introduction}

Flavin mononucleotide (FMN) is non-covalently bound to complex I of the mitochondrial respiratory chain. During ischemia-reperfusion injury, reduced FMN is released from complex I, leaving the complex impaired. In literature, FMN measured during ex situ organ perfusion has been considered as a biomarker for organ graft quality. With this protocol the FMN concentrations in perfusate samples taken during organ perfusion can be estimated quickly and easily by fluorescence spectrometry. This assay will work for samples acquired from whole blood, red cell based solutions or acellular solutions.

\section{Reagents}

· Riboflavin 5'-monophosphate sodium salt hydrate (FMN) from Sigma-Aldrich (F6750-5G)

Sodium chloride $(\mathrm{NaCl})$ 0.9\% Viaflo from Baxter SA

\section{Equipment}

Black nonbinding 96-well microplate from Greiner Bio-one (655900)

Plate reader for fluorescence intensity: FLUOstar Omega from BMG Labtech

\section{Procedure}

\section{A. Prepare a standard curve of known concentrations of FMN}

1. Dissolve $\mathrm{FMN}$ in $\mathrm{NaCl} 0.9 \%$ at a concentration of $6.25 \mathrm{mg} / \mathrm{ml}$ 
2. Mix thoroughly by vortex

3. Prepare a serial dilution in $0.9 \%$ sodium chloride to obtain $F M N$ solutions ranging from $9.8 \mathrm{ng} / \mathrm{ml}$ to $625 \mathrm{ng} / \mathrm{ml}$

a. $\quad 62.5 \mu \mathrm{g} / \mathrm{ml}$ : Ad $10 \mu \mathrm{l}$ stock to $990 \mu \mathrm{l} \mathrm{NaCl} 0.9 \%$; mix thoroughly by vortex

b. $625 \mathrm{ng} / \mathrm{ml}$ : Ad $100 \mu \mathrm{l}$ a. to $9900 \mu \mathrm{l} \mathrm{NaCl} 0.9 \%$; mix thoroughly by vortex

c. $312.5 \mathrm{ng} / \mathrm{ml}: 1000 \mu \mathrm{l}$ b. to $1000 \mu \mathrm{l} \mathrm{NaCl} 0.9 \%$; mix thoroughly by vortex

d. $156.3 \mathrm{ng} / \mathrm{ml}: 1000 \mu \mathrm{l}$ c. to $1000 \mu \mathrm{l} \mathrm{NaCl} 0.9 \%$; mix thoroughly by vortex

e. $\quad 78.1 \mathrm{ng} / \mathrm{ml}: 1000 \mu \mathrm{ld}$. to $1000 \mu \mathrm{l} \mathrm{NaCl} 0.9 \%$; mix thoroughly by vortex

f. $\quad 39.1 \mathrm{ng} / \mathrm{ml}: 1000 \mu \mathrm{l}$ e. to $1000 \mu \mathrm{l} \mathrm{NaCl} 0.9 \%$; mix thoroughly by vortex

g. $\quad 19.5 \mathrm{ng} / \mathrm{ml}: 1000 \mu \mathrm{l}$ f. to $1000 \mu \mathrm{l} \mathrm{NaCl} 0.9 \%$; mix thoroughly by vortex

h. $9.8 \mathrm{ng} / \mathrm{ml}: 1000 \mu \mathrm{l}$ g. to $1000 \mu \mathrm{NaCl} 0.9 \%$; mix thoroughly by vortex

4. Load $100 \mu \mathrm{l}$ of each FMN solution (b. to h.) into a black nonbinding 96 -well microplate well in eightfold

5. Load $100 \mu \mathrm{l} 0.9 \% \mathrm{NaCl}$ into a black nonbinding 96 -well microplate well in eightfold as blank

6. Measure fluorescence intensity using an excitation filter of $485 \mathrm{~nm}$ and an emission filter of $520 \mathrm{~nm}$ with top optics and gain adjustment on well containing the highest concentration $(625 \mathrm{ng} / \mathrm{ml})$

7. Construct a linear standard curve:

a. Calculate mean fluorescence of each FMN solution and the blank 
b. Calculate the standard deviation of each FMN solution and the blank

c. Calculate the \%CV ((standard deviation/mean)*100) of each FMN solution and the blank; this must be less than $10 \%$ (usually less than $2 \%$ )

d. Subtract the mean of the blank from the mean of the different FMN solutions

e. Prepare a linear standard curve using the data produced from the diluted FMN solutions. Put 'mean fluorescence' - 'mean blank' on the Y-axis (linear) and concentration ( $\mathrm{ng} / \mathrm{ml}$ ) on the X-axis (linear)

\section{B. Measure fluorescence in perfusate samples}

1. Preparation of perfusate samples

a. Collect samples at different time points during the experiment in EDTA tubes

b. Centrifuge at $3500 \mathrm{rpm}$ at $4^{\circ} \mathrm{C}$ for 10 minutes within 20 minutes after collection

c. Pipet the supernatant in a tube and immediately freeze in liquid nitrogen

d. Store at $-80^{\circ} \mathrm{C}$ until use

2. Preparation of positive control samples

a. As controls, pool samples with expected low and high amounts of FMN together. These samples should ideally be from the same set of experiments or at least from experiments with the same perfusate composition, so they have the same matrix

b. Store aliquots at $-80^{\circ} \mathrm{C}$

3. Measure FMN concentration in unknown perfusate samples

a. Load $100 \mu$ l of perfusate sample into a black nonbinding 96 -well microplate well in duplicate 
b. Load $100 \mu \mathrm{l}$ of $\mathrm{NaCl} 0.9 \%$ into the same black nonbinding 96 -well microplate in eightfold as blank

c. Load $100 \mu$ l of the low and high controls into the same black nonbinding 96-well microplate in eightfold as positive control

d. Measure fluorescence intensity using an excitation filter of $485 \mathrm{~nm}$ and an emission filter of $520 \mathrm{~nm}$ with top optics and gain adjustment set by the standard curve plate run earlier that day

e. Calculate mean fluorescence of each perfusate sample, controls and the blank

f. Calculate the standard deviation of each perfusate sample, controls and the blank

g. Calculate the \%CV ((standard deviation/mean)*100) of each perfusate sample, control and the blank; this must be less than $10 \%$ (usually less than $2 \%$ )

h. Subtract the mean of the blank from the mean of the different perfusate samples and the controls

i. Extrapolate the sample and control FMN concentration $(\mathrm{ng} / \mathrm{ml})$ from the standard curve

j. The intra-assay variance for the controls must be less than $10 \%$

k. Perform a weight correction for the unknown perfusate samples $(\mathrm{ng} / \mathrm{ml} / 100 \mathrm{~g})$

Data on 'Expected results' and 'Intra-assay variance' can be found in the supplementary files.

\section{Troubleshooting}

1. Fluorescence intensity of the blank higher than the fluorescence intensity of the $9.8 \mathrm{ng} / \mathrm{ml} \mathrm{FMN}$ solution

a. Use nonbinding microplates

2. Poor standard curve

a. Use freshly prepared FMN solutions

b. Confirm correct dilutions

c. Confirm if standard is properly dissolved 
3. Calculated concentration of the positive control not in the expected range

a. Confirm use of correct microplate

b. Confirm calculations

\section{Supplementary Files}

This is a list of supplementary files associated with this preprint. Click to download.

- SupplementaryfilesFMNprotocol.docx 